\title{
K-haler breath-triggered inhaler: a profile of the properties of the device
}

\author{
Emma D. Deeks ${ }^{1} \cdot$ Katherine A. Lyseng-Williamson $^{1}$ (i)
}

Published online: 6 May 2019

๑) Springer Nature 2019, corrected publication 2019

\begin{abstract}
The k-haler ${ }^{\circledR}$ device is a new breath-triggered pressurized metered-dose inhaler (BTI). Its breath-triggered mechanism employs unique 'kinked-hose valve' technology ( $\mathrm{k}$-valve $\left.{ }^{\mathrm{TM}}\right)$ and is easy to actuate. In vitro, the k-haler device required less respiratory power, which is affected by the resistance of the device, for dose release than a dry powder inhaler (DPI), potentially making it suitable for a wider range of patients, including those with a low inspiratory flow rate. It has a lower, more consistent plume force than conventional press-and-breathe pressurized metered-dose inhalers (pMDIs), has high fineparticle fraction and pulmonary deposition rates, and, in contrast to conventional pMDIs (but like other BTIs and DPIs), does not require actuation-inhalation coordination. The k-haler device is compact, has an easy-to-read dose counter, a connected mouthpiece and is simple to use, with limited data indicating that more patients are satisfied with the device than they are with some DPIs and that many patients prefer it over some commonly used inhalers. To facilitate correct use, the packaging of the k-haler contains simple how-to-use instructions and diagrams, as well as a patient information leaflet. A number of inhaled products may be suitable for administration via the k-haler device.
\end{abstract}

\section{Adis evaluation of the $k$-haler device}

in obstructive lung disorders

Breath-triggered pressurized metered-dose inhaler that releases drug dose via unique 'kinked-hose valve' technology (k-valve $\left.{ }^{\mathrm{TM}}\right)$

Simple to use/actuate; requires less inspiratory power for dose release than a dry-powder inhaler

Lower, more consistent plume force than a conventional pressurized metered-dose inhaler

High fine-particle fraction and pulmonary deposition rates

Largely preferred over some commonly used inhalers
Katherine A. Lyseng-Williamson

dtp@adis.com

1 Springer, Private Bag 65901, Mairangi Bay, Auckland 0754, New Zealand

\section{What is the rationale for developing the $\mathrm{k}$-haler device?}

Achieving good symptom control and minimizing the risk of exacerbations are among the long-term goals of managing obstructive lung disorders [1]. Medications, such as inhaled corticosteroids (ICSs) and long-acting $\beta_{2}$-agonists (LABAs), used in the management of obstructive lung disorders are generally delivered to the lungs via inhalers, with the importance of correct inhaler technique being recognized as key for therapeutic benefit $[1,2]$. However, patients often make mistakes when administering their medication [3-7], which increases the likelihood of poor treatment outcomes [5-7]. Predominant inhaler types include dry powder inhalers (DPIs) and conventional press-and-breathe pressurized metered-dose inhalers (pMDIs) [8]. These devices require different techniques to optimize pulmonary drug delivery, with DPIs being passive devices that need forceful inhalation for drug extraction and disaggregation, and pMDIs being active devices that use propellant for drug expulsion and require actuation and inhalation to be coordinated [8]. However, in a recent observational study [7], insufficient respiratory effort and poor coordination between actuation and inhalation were identified as critical errors (i.e. associated with poor outcomes) for DPIs and pMDIs, respectively, 
highlighting the need for alternative device options, one of which is the breath-triggered pMDI (BTI).

BTIs are similar to conventional pMDIs in that they use propellant to generate respirable drug particles, but are similar to DPIs in that actuation occurs as a patient breathes in $[8,9]$. Only a low respiratory flow rate is required for BTI actuation (in contrast to DPIs) and there is no need for actuation and breathing to be coordinated (unlike conventional pMDIs) [9]. Consequently, patients seem to prefer BTIs over other inhaler devices and find them easier to use correctly than conventional pMDIs [10, 11].

The most recent BTI is the ${\mathrm{k}-h a l e{ }^{\circledR}}^{\circledR}$ device. It is currently available for administering a fixed-dose combination of an ICS (fluticasone propionate) + a LABA (formoterol fumarate), which has previously only being available in a conventional pMDI device. This combination is marketed as the flutiform ${ }^{\circledR} \mathrm{k}$-haler in the $\mathrm{EU}$, and is approved for the regular treatment of asthma for which an ICS + LABA combination is appropriate [12].

\section{What are the characteristics of the k-haler device?}

The k-haler device is a small and compact inhaler that uses 'kinked-hose valve' technology, known as 'k-valve ${ }^{\mathrm{TM}}$ ', for dose release (Fig. 1) [8]. When the device is primed for use (i.e. shaken and manually actuated four times prior to the first use of the inhaler) [12], the drug enters the k-valve and is held at the tube's kink (Fig. 1a) until the patient inhales, an action that straightens the k-valve and results in the drug dose being released (Fig. 1b) [8].

The k-haler device has a similar shape to that of a conventional pMDI (Fig. 1c) and requires few steps to operate $[8,12]$ :

- shake (to mix contents, while exhaling slowly and deeply);

- open the mouthpiece (while holding the device upright);

- inhale (slowly and deeply);

- close (while holding breath for as long as possible).

\section{Drug delivery properties}

When the plume spray force of flutiform k-haler was compared with that of two ICS/LABA conventional pMDIs, the k-haler device had the lowest spray force, with the greatest difference $(\approx 70-87 \%)$ evident at distances of $60-95 \mathrm{~mm}$, which is typical of the distance between an inhaler mouthpiece and the back of the throat [13]. Compared with the pMDIs, the k-haler device also had a more consistent plume force, which may in part be because it is a BTI and thus has none of the actuation speed variability of press-and-breathe devices. Differences in the properties of the device propellants may also have contributed to the observed difference in plume force. For instance, the boiling point of the k-haler device propellant (apaflurane HFA-227; $-16.4{ }^{\circ} \mathrm{C}$ ) is higher than that of the propellant of some other pMDIs (norflurane HFA-134a; $-26.5^{\circ} \mathrm{C}$ ), with resultant differences in evaporation rate and thus, likely, plume force [13]. (a)

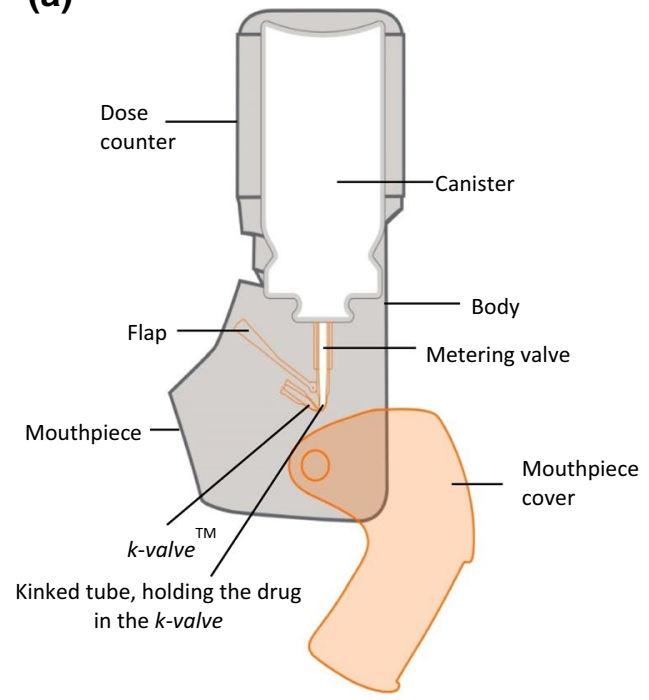

(b)

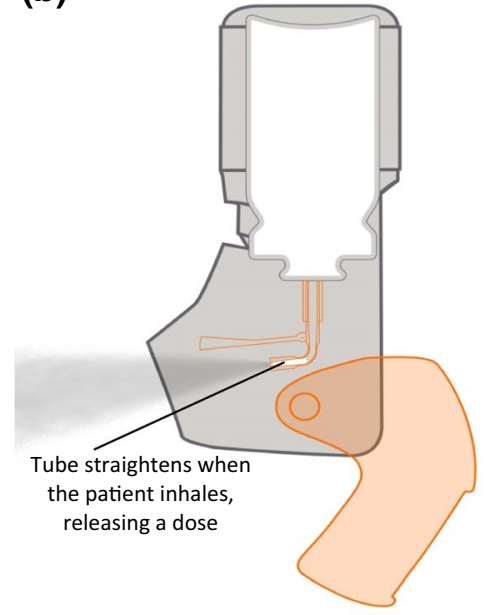

(c)

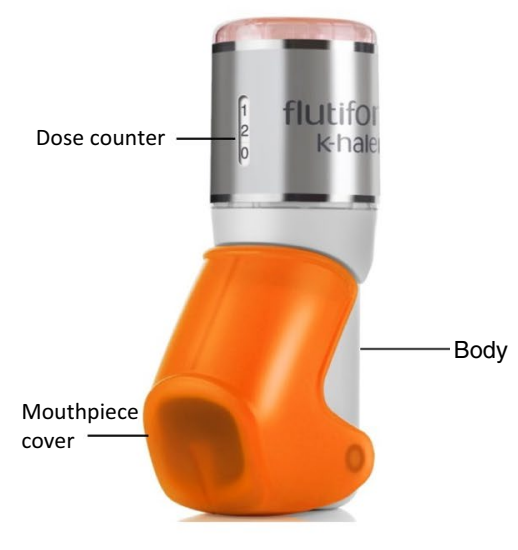

Fig. 1 Interior and exterior components of the k-haler ${ }^{\circledR}$ device, which uses kinked-hose technology ( $\mathrm{k}$-valve ${ }^{\mathrm{TM}}$ ) as a breath-trigger mechanism. a Priming the device releases the drug into the k-valve where is held by the kinked positioning of the tube and $\mathbf{b}$ inhalation by the patient straights the tube, thereby releasing the dose; $\mathbf{c}$ external appearance of the k-haler (Reproduced and adapted with permission from Mundipharma) 
The particle size and lung deposition of fluticasone and formoterol delivered via the flutiform k-haler [14] are comparable to those delivered by the flutiform (fluticasone propionate + formoterol fumarate) pMDI [15]. A high proportion $(\approx 40 \%)$ of a fluticasone and formoterol dose are delivered as fine particles (i.e. $<5 \mu \mathrm{m}$ ) in vitro) with the k-haler device. This property, in addition to its favourable plume force, may be key factors with regards to the high pulmonary drug deposition rates shown with the flutiform k-haler. In a single-dose, open-label, scintigraphic study of the k-haler device, mean pulmonary deposition of fluticasone and formoterol was $27 \%$ of a delivered dose and $26 \%$ of a metered dose in 12 healthy adults; corresponding results in 12 adults with asthma were 45 and 43\% [14]. The unexpected relative low lung deposition in healthy adults may have been due to undetected errors in using the k-haler. Mean penetration indices (i.e. peripheral:central ratio) with the k-haler were 0.44 and 0.31 in healthy and asthmatic adults, respectively, indicating that almost a quarter of the administered dose reached the lung periphery in the asthma population with the slightly higher index in healthy individuals likely being due to their normal and pathology-free airways [14].

The type of inhaler (i.e. k-haler vs conventional pMDI $\pm \mathrm{a}$ spacer) does not appear to affect the pulmonary and systemic bioavailability of fluticasone + formoterol (administered as fixed-dose combinations) to a clinically relevant extent, according to the results of single-dose pharmacological studies in 35-48 healthy volunteers $[16,17]$.

\section{Physical properties of the exterior of the inhaler}

The mouthpiece cap is connected to the k-haler, making it easy to re-position and impossible to lose, and is orange in colour, facilitating its identification and correct device orientation for use (Fig. 1c) [8, 12]. If a primed dose is not taken, the dose is automatically released into the mouthpiece cap when the cap is closed, both signalling that the dose was not taken and preventing double-dosing [8].

On the front of the device is a single-digit colour-coded dose counter in an easy-to-read font (designed by the UK Royal National Institute for the Blind), allowing the number of doses remaining in the inhaler to be seen (Fig. 1c). In addition, to make it easy for patients to know when a new prescription is needed, one red bar appears in the dose counter when there are $<30$ doses remaining, which changes to two red bars when the device is empty [8].

\section{Is the k-haler device simple to use?}

It is easy to learn how to use the k-haler device, as shown by the results of two randomized, open-label, two-period crossover studies in adult patients with obstructive lung disease $[18,19])$.

The ability to correctly handle the flutiform k-haler was similar to that of the flutiform pMDI in 307 patients (66 aged 12-17 years, 166 aged 18-65 years and 75 aged $>65$ years) in one study [18]. The proportion of patients able to perform all steps correctly at the first attempt (primary endpoint) was identical (77.2\%) with both inhalers, with 87.0 and $82.4 \%$ of patients being able to perform all critical steps correctly at the first attempt with the k-haler and pMDI, respectively. Almost all patients (99.7 and 99.3\%) were trained to use the k-haler and pMDI within $15 \mathrm{~min}$, and only a few (7 and 9\%) required more than two attempts to master usage of the respective inhalers [18].

In the other study, 362 patients were better able to use the flutiform k-haler correctly than the budesonide/formoterol DPI (Symbicort ${ }^{\circledR}$ Turbuhaler $^{\circledR}$ ) at the end of a 12-week period [19]. At the end of week 12, a significantly $(p<0.001)$ higher proportion of patients could perform all critical handling steps with the k-haler than with the DPI [94.0 vs 82.4\%; odds ratio 2.89 (95\% CI 1.57-5.33); primary endpoint]. Likewise, the proportion of patients who could perform all steps correctly was significantly $(p<0.001)$ higher with the k-haler than with the DPI [73.7 vs $61.6 \%$; odds ratio 1.85 (95\% CI 1.30-2.62)] [19].

\section{Compared with the flutiform pMDI and usual maintenance inhalers}

The handling profile of the flutiform k-haler was similar to that of the flutiform pMDI [18]. After being trained, most patients correctly performed all handling steps (77.2\%; primary endpoint) and all critical handling steps $(87.0 \%)$ at first attempt for flutiform k-haler, and virtually all patients (99.7\%) were able to perform all handling steps correctly only 15 min after training, regardless of their age or lung function [18]. For the flutiform pMDI, the respective rates were similar $(77.2,82.4$ and $99.3 \%$, respectively) [18].

Moreover, patients preferred the flutiform k-haler over their usual maintenance ICS/LABA inhaler according to a post hoc analysis of 288 patients using specific maintenance DPIs [i.e. Symbicort Turbuhaler (budesonide/ formoterol); Seretide ${ }^{\circledR}$ Accuhaler ${ }^{\circledR}$ (fluticasone/salmeterol)] or pMDIs [i.e. Seretide ${ }^{\circledR}$ Evohaler $^{\circledR}$ (fluticasone/ salmeterol); Fostair ${ }^{\circledR}$ (beclomethasone/formoterol pMDI)] [18]. A large proportion of patients (61-71\%) preferred the 
flutiform k-haler over their usual maintenance inhaler, with $11-24 \%$ having no preference, and $11-20 \%$ preferring their usual inhaler. Most patients found the k-haler device easier to use than their usual DPI (62 and 63\% of Turbuhaler and Accuhaler users), with 17 and $26 \%$ finding the ease-of- use about the same, and 21 and $11 \%$ finding their usual DPI easier to use. Relative to the use of their usual pMDI, most patients found the ease-of-use of the k-haler device to be either better (58 and $44 \%$ of Evohaler and Fostair users) or about the same (24 and $44 \%$ ); only 17 and $11 \%$ found their usual pMDI easier to use.

In contrast, only $37-49 \%$ of patients preferred the flutiform pMDI over their usual maintenance inhaler, 25-56\% had no preference, and 0-29\% preferred their usual inhaler. The flutiform pMDI was easier to use than their usual inhaler according to 29, 29, 28 and $0 \%$ of Turbuhaler, Accuhaler, Evohaler and Fostair users, with most (49, 57, 69 and 89\%) finding the ease-of-use about the same, and few (21, 14, 3 and $11 \%$ ) finding their usual inhaler easier to use [18].

\section{Compared with the budesonide/formoterol DPI}

Patients found it easier to learn how to use the flutiform k-haler than the budesonide/formoterol DPI (Turbuhaler) [19]. For example, 12 weeks after being trained, significantly $(p<0.001)$ more patients in the flutiform k-haler group than in the budesonide/formoterol DPI group were able to correctly perform all critical device-handling steps (94.0 vs $82.4 \%$; primary endpoint) and all handling steps (73.7 vs $61.6 \%$ ). Moreover, on day 1 , the proportion of patients being able to correctly perform the critical handling steps using only the 'instructions for use' as guidance (i.e. without training) was significantly higher in the flutiform k-haler group than in the budesonide/formoterol DPI group (72.7 vs $36.1 \% ; p<0.001)$ [19].

Consistent with these findings, significantly $(p<0.001)$ more patients were satisfied with the flutiform k-haler than with the budesonide/formoterol DPI for all aspects evaluated, among which were appearance, opening/preparation, knowing when a dose has been received, knowing how many doses are left and overall ease of use [19].

\section{Is the k-haler device easy to actuate?}

The k-haler device is easy to actuate, with its dose-release mechanism being triggered on first attempt by almost all (99\%) patients with obstructive lung disease in the randomized crossover trial [18]. Those who failed to trigger actuation had lung disease of varying severity, suggesting the issue may have been poor technique rather than insufficient inspiratory flow rate [18].
In an in vitro comparison, the average inspiratory flow rate needed to trigger was $20-40 \mathrm{~L} / \mathrm{min}$ for the $\mathrm{k}$-haler BTI, Easi-Breathe ${ }^{\circledR}$ BTI and NEXThaler ${ }^{\circledR}$ DPI, although it was significantly $(p<0.005)$ lower with the Easi-Breathe BTI than with either of the other two inhalers [20]. However, the average respiratory power, which is affected by the resistance of the device, needed to trigger the two BTIs (k-haler and Easi-Breathe) was significantly $(p<0.001)$ less than that needed for the DPI (NEXThaler) [0.1 and 0.07 vs $0.55 \mathrm{~W}$; values estimated from graph] [20].

\section{What tools are available to help patients learn to use the k-haler correctly?}

Simple instructions for using the k-haler device feature on the packaging of flutiform k-haler (Fig. 2) as well as in the patient information leaflet (PIL) [8]. The PIL also includes an internet link to additional instructions and support to help patients learn and maintain correct inhaler technique, including a training film and an interactive training tool [8].

\section{What conclusions can be made regarding the k-haler device?}

The technology and packaging of the k-haler device addresses some of the challenges of traditional inhaler use. The device received the A' Design Platinum Awards for 'Scientific Instruments, Medical Devices \& Research Equipment Devices' and 'Ease of Use and Universal Design' in 2017-2018 [21] and was the gold winner of the SPARK design award in 2012 [22].

Using a BTI, such as the k-haler device, rather than a DPI or conventional pMDI to administer medications for

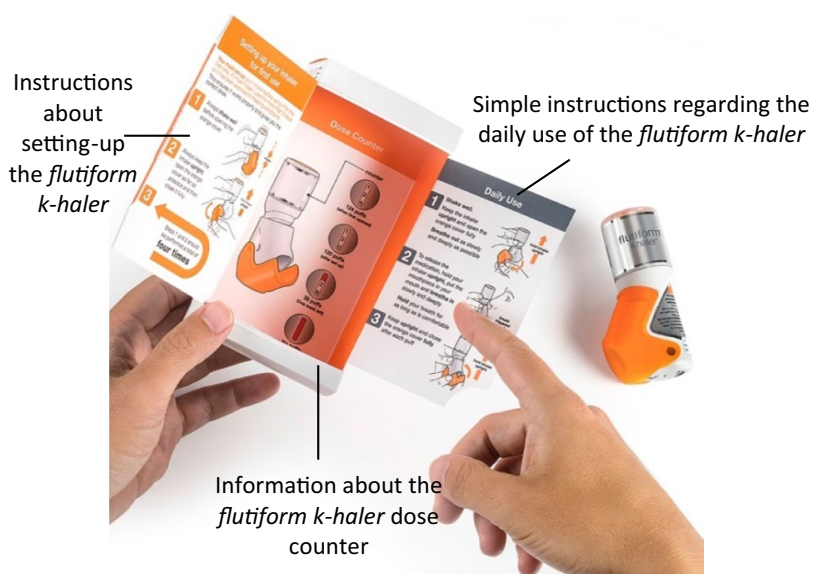

Fig. 2 Flutiform ${ }^{\circledR}$ k-haler ${ }^{\circledR}$ informative packaging (Reproduced and adapted with permission from Mundipharma) 
obstructive lung disorders has the benefits of requiring neither forcible inhalation nor physical coordination of actuation and inhalation. The breath-trigger mechanism in the k-haler device employs 'kinked-hose valve' technology and is simple to actuate, requiring less inspiratory power for dose release than a DPI. This potentially makes the $\mathrm{k}$-haler device suitable for a wide range of patients, including those whose inspiratory rate is low. It has a lower, more consistent plume force than conventional pMDIs, has a high fine-particle fraction and pulmonary deposition and, in contrast to conventional pMDIs (but like other BTIs and DPIs), does not require actuation-inhalation coordination.

The k-haler device is compact, has an easy-to-read dose counter and a connected mouthpiece, and is simple to use, with limited data indicating that more patients are satisfied with the device than they are with some DPIs, and that many patients prefer it over some commonly used inhalers.

Consideration has also been given to the packaging of flutiform k-haler, which contains simple instructions and diagrams on how to use the device correctly. As PILs can be complicated and confusing for patients (resulting in some patients not reading them fully and missing key usage information) [23], providing simple how-to-use information on the packaging (in addition to a PIL) may facilitate correct inhaler technique.

The k-haler device is currently available for administering fluticasone/formoterol (flutiform k-haler), making it the first BTI for administering a fixed-dose combination of an ICS + a LABA in Europe. Although other BTI devices are available in Europe (i.e. the Autohaler ${ }^{\circledR}[24]$ and EasiBreathe [25] devices), none of the formulations that use these devices co-deliver an ICS + a LABA. In addition to fluticasone/formoterol, a range of other inhaled drugs may also be suitable for administration via the k-haler device.

Acknowledgements Reviewers of the manuscript include: Z. Teitelbaum, Medicinal Chemistry Research Division, Israel Institute for Biological Research, Ness Ziona, Israel. During the peer review process, Mundipharma, the marketing-authorization holder of the k-haler ${ }^{\circledR}$, was also offered an opportunity to provide a scientific accuracy review of their data. Changes resulting from comments received were made on the basis of scientific and editorial merit.

\section{Compliance with ethical standards}

Funding The preparation of this review was not supported by any external funding.

Conflict of interest E.D. Deeks and K. A. Lyseng-Williamson are employees of Adis/Springer, are responsible for the article content and declare no conflicts of interest.

Open Access This article is distributed under the terms of the Creative Commons Attribution-NonCommercial 4.0 International License (http://creativecommons.org/licenses/by-nc/4.0/), which permits any noncommercial use, duplication, adaptation, distribution and reproduction in any medium or format, as long as you give appropriate credit to the original author(s) and the source, provide a link to the Creative Commons license and indicate if changes were made.

\section{References}

1. Global strategy for asthma management and prevention: 2019 update. Bethesda: Global Initiative for Asthma; 2019.

2. Makela MJ, Backer V, Hedegaard M, et al. Adherence to inhaled therapies, health outcomes and costs in patients with asthma and COPD. Respir Med. 2013;107(10):1481-90.

3. Al Ammari M, Sultana K, Yunus F, et al. A cross-sectional observational study to assess inhaler technique in Saudi hospitalized patients with asthma and chronic obstructive pulmonary disease. Saudi Med J. 2016;37(5):570-4.

4. Basheti IA, Obeidat NM, Ammari WG, et al. Associations between inhaler technique and asthma control among asthma patients using pressurised MDIs and DPIs. Int J Tuberc Lung Dis. 2016;20(5):689-95.

5. Al-Jahdali H, Ahmed A, Al-Harbi A, et al. Improper inhaler technique is associated with poor asthma control and frequent emergency department visits. Allergy Asthma Clin Immunol. 2013;9(1):8.

6. Maricoto T, Rodrigues LV, Teixeira G, et al. Assessment of inhalation technique in clinical and functional control of asthma and chronic obstructive pulmonary disease. Acta Med Port. 2015;28(6):702-7.

7. Price DB, Román-Rodríguez M, McQueen RB, et al. Inhaler errors in the CRITIKAL study: type, frequency, and association with asthma outcomes. J Allergy Clin Immunol Pract. 2017;5(4):1071-81.

8. Data on file. Cambridge: Mundipharma International Limited; 2018.

9. Rees J. Methods of delivering drugs. BMJ. 2005;331(7515):504-6.

10. Lenney J, Innes JA, Crompton GK. Inappropriate inhaler use: assessment of use and patient preference of seven inhalation devices. Respir Med. 2000;94(5):496-500.

11. Price DB, Pearce L, Powell SR, et al. Handling and acceptability of the Easi-Breathe device compared with a conventional metered dose inhaler by patients and practice nurses. Int J Clin Pract. 1999;53(1):31-6.

12. Flutiform K-haler (fluticasone propionate/formoterol fumarate dihydrate $50 \mathrm{mcg} / 5 \mathrm{mcg}$ and $125 \mathrm{mcg} / 5 \mathrm{mcg}$ per actuation pressurised inhalation, suspension): summary of product information. Cambridge: Napp Pharmaceuticals; 2018.

13. Tuohy J, Marshall J, Danagher H. Plume spray force of three HFA-propelled ICS/LABA combination inhalers [abstract no.4655 + poster]. In: European Respiratory Society Congress; 2017.

14. Kappeler D, Sommerer K, Kietzig C, et al. Pulmonary deposition of fluticasone propionate/formoterol in healthy volunteers, asthmatics and COPD patients with a novel breath-triggered inhaler. Respir Med. 2018;138:107-14.

15. Johal B, Howald M, Fischer M, et al. Fine particle profile of fluticasone propionate/formoterol fumarate versus other combination products: the DIFFUSE study. Comb Prod Ther. 2013;3:39-51.

16. Bell D, Mundin G, Lomax M, et al. Relative pulmonary bioavailability (BA) of fluticasone propionate/formoterol (FP/FORM) via pressurised metered-dose inhaler (pMDI) and a novel breath-triggered inhaler (BTI) [abstract no. $4618+$ poster]. In: European Respiratory Society Congress; 2017.

17. Bell D, Mundin G, Lomax M, et al. Systemic bioavailability (BA) and pharmacodynamics (PD) of fluticasone propionate/formoterol (FP/FORM) via pressurised metered-dose inhaler (pMDI) or a 
novel breath-triggered inhaler (BTI) [abstract no. $4633+$ poster]. In: European Respiratory Society Congress; 2017.

18. Bell D, Mansfield L, Lomax M. A randomized, crossover trial evaluating patient handling, preference, and ease of use of the fluticasone propionate/formoterol breath-triggered inhaler. J Aerosol Med Pulm Drug Deliv. 2017;30(6):425-43.

19. Bell D, Timmer W, Mansfield L, et al. Patients' ability to use a novel fluticasone propionate/formoterol breath-triggered inhaler (BTI) vs. budesonide/formoterol dry powder inhaler (DPI) [abstract no. $9732+$ poster]. In: European Respiratory Society Congress; 2017.

20. Bischofberger JE, Rhima CE, Tibbatts J, et al. A study on the inspiratory flow rate and power required to trigger flutiform ${ }^{\circledR}$ k-haler ${ }^{\circledR}$, Qvar ${ }^{\circledR}$ Easie-Breathe ${ }^{\circledR}$, and Fostair ${ }^{\circledR}$ NEXThaler ${ }^{\circledR}$ breath actuated inhalers [abstract + poster]. In: Respiratory drug delivery conference; 2018.

21. A' Design Award \& Competition. 'Scientific Instruments, Medical Devices and Research Equipment Design Award' 2017-2018 period winners and 'Ease of Use and Universal Design Awards' 2017-2018 period winners. 2017. https://competition.adesignawa rd.com/winners.php. Accessed 25 Mar 2019.

22. Spark. K-haler. Winner-gold. 2012. http://www.sparkawards.com/ galleries/index.cfm?entry=1DD7D361-0D98-03C9-7058504DE3 B54B93. Accessed 25 Mar 2019.

23. van Dijk L, Monteiro SP, Vervloet M, et al. Study on the packjage leaflets and the summaries of product characteristics of medicinal products for human use. PIL-S study. 2014. http://ec.europa.eu/ health/files/committee/75meeting/pil_s.pdf. Accessed 25 Mar 2019.

24. ADMIT Inhalers 4u. Breath-actuated MDI: Autohaler. https:// www.inhalers4u.org/index.php/autohaler/. Accessed 28 Mar 2019.

25. ADMIT Inhalers $4 \mathrm{u}$. Breath-actuated MDI: Easi Breath. https:// www.inhalers4u.org/index.php/easi-breathe/. Accessed $28 \mathrm{Mar}$ 2019. 